\title{
Existence and multiplicity of periodic solutions generated by impulses
}

Liu Yang*

\section{"Correspondence:}

yangliuyanzi@163.com

Department of Mathematics and

Computing Sciences, Hengyang

Normal University, Hengyang,

Hunan 421008, People's Republic of China

\begin{abstract}
In this paper, we investigate the existence and multiplicity of periodic solutions for a class of $p$-Laplacian systems with impulses. By using variational methods and critical point theory, we obtain that such a system possesses at least one, two periodic solutions generated by impulses under different conditions, respectively. Recent results in the literature are generalized and significantly improved.
\end{abstract}

Keywords: periodic solutions; solution generated by impulses; critical point

\section{Introduction}

Consider the following $p$-Laplacian system with impulsive condition:

$$
\left\{\begin{array}{l}
\left(\left|u^{\prime}(t)\right|^{p-2} u^{\prime}(t)\right)^{\prime}+V_{u}(t, u(t))=\mu F_{u}(t, u(t)), \quad t \in\left(s_{k-1}, s_{k}\right) \\
\Delta\left(\left|u^{\prime}\left(s_{k}\right)\right|^{p-2} u^{\prime}\left(s_{k}\right)\right)=g_{k}\left(u\left(s_{k}\right)\right), \\
u(0)-u(T)=u^{\prime}(0)-u^{\prime}(T)=0,
\end{array}\right.
$$

where $p>1, s_{k}, k=1,2, \ldots, m$, are instants in which the impulses occur and $0=s_{0}<s_{1}<s_{2}<$ $\cdots<s_{m}<s_{m+1}=T, \Delta u^{\prime}\left(s_{k}\right)=u^{\prime}\left(s_{k}^{+}\right)-u^{\prime}\left(s_{k}^{-}\right)$with $u^{\prime}\left(s_{k}^{ \pm}\right)=\lim _{t \rightarrow s_{k}^{ \pm}} u^{\prime}(t), g_{k}(u)=\operatorname{grad}_{u} G_{k}(u)$, $G_{k} \in C^{1}\left(\mathbb{R}^{N}, \mathbb{R}\right), V \in C^{1}\left([0, T] \times \mathbb{R}^{N}, \mathbb{R}\right), V_{u}(t, u(t))=\operatorname{grad}_{u} V(t, u), F \in C^{1}\left([0, T] \times \mathbb{R}^{N}, \mathbb{R}\right)$, $F_{u}(t, u(t))=\operatorname{grad}_{u} F(t, u), \mu \geq 0$ is a parameter.

Impulsive differential equations can be used to describe the dynamics of processes which possess abrupt changes at certain instants. Up to now, impulsive differential systems have been widely applied in many science fields such as control theory, biology, mechanics and so on; see [1-7] and references therein. For general theory of impulsive differential equations, we refer the readers to the monographs as [8-10].

The existence of solutions is one of the most important topics of impulsive differential systems. Many classical methods and tools, such as coincidence degree theory, fixed point theory and the method of upper and lower solutions, have been used to study them; see [11-15] and references therein.

Recently, some authors creatively applied the variational method to deal with impulsive problems, see [16-21]. The variational method is opening a new approach to dealing with discontinuity problems such as impulses. However, when the problems studied in [1621] degenerate to the cases without impulses, plenty of the corresponding results can also be obtained under the same conditions. Therefore, the effect of impulses was not seen

(c2013 Yang; licensee Springer. This is an Open Access article distributed under the terms of the Creative Commons Attribution License (http://creativecommons.org/licenses/by/2.0), which permits unrestricted use, distribution, and reproduction in any medium, provided the original work is properly cited. 
evidently. Due to this point, in [22-24], the existence of solutions generated by impulses is investigated.

Definition 1.1 ([22]) A solution is called a solution generated by impulses if this solution is non-trivial when impulsive terms are not zero, but it is trivial when impulsive term is zero.

For example, if problem $\left(P_{p, \mu}\right)$ does not possess a non-zero solution when $g_{k} \equiv 0$ for all $1 \leq k \leq m$, then the non-zero solution for problem $\left(P_{p, \mu}\right)$ is called a solution generated by impulses. In detail, Zhang and $\mathrm{Li}$ [22] obtained the following theorem.

Theorem A ([22]) Assume that $V, W$ satisfy the following conditions:

$\left(\mathrm{V}_{1}\right) V$ is continuously differentiable and there exist positive constants $b_{1}, b_{2}>0$ such that $b_{1}|u|^{2} \leq-V(t, u) \leq b_{2}|u|^{2}$ for all $(t, u) \in[0, T] \times \mathbb{R}^{N} ;$

$\left(\mathrm{V}_{2}\right)-V(t, u) \leq-V_{u}(t, u) u \leq-2 V(t, u)$ for all $(t, u) \in[0, T] \times \mathbb{R}^{N}$;

(g. There exists $\theta>2$ such that $g_{k}(u) u \leq \theta G_{k}(u)<0$ for all $u \in \mathbb{R}^{N} \backslash\{0\}$ and $k=1,2, \ldots, m$.

Then the problem

$$
\left\{\begin{array}{l}
u^{\prime \prime}(t)+V_{u}(t, u(t))=0, \quad t \in\left(s_{k-1}, s_{k}\right), \\
\Delta\left(u^{\prime}\left(s_{k}\right)\right)=g_{k}\left(u\left(s_{k}\right)\right), \\
u(0)-u(T)=u^{\prime}(0)-u^{\prime}(T)=0,
\end{array}\right.
$$

possesses at least one non-zero solution generated by impulses.

For $p$-Laplacian systems with impulsive condition, there are many excellent results by using the variational method, see [24-26]. In particular, Han et al. [24] obtained the existence of solutions for $p$-Laplacian systems generated by impulses.

Motivated by the facts mentioned above, in this paper, we further study the existence of a solution for problem $\left(P_{p, \mu}\right)$ generated by impulses under more general conditions. In addition, we investigate the multiple solutions generated by impulses. First, we give the following new definition of the solution generated by impulses.

Definition 1.2 A solution for problem $\left(P_{p, \mu}\right)$ is said to be generated by impulses if this solution emerges when impulsive terms are not zero, but disappears when impulsive terms are zero.

Remark 1.3 Definition 1.2 contains Definition 1.1. In addition, if problem $\left(P_{p, \mu}\right)$ possesses at most one solution when $g_{k} \equiv 0$ for all $1 \leq k \leq m$, but it possesses three solutions when impulsive terms are not zero, then problem $\left(P_{p, \mu}\right)$ has at least two solutions generated by impulses.

Now, we state our results.

Theorem 1.4 Assume that $V, G$ satisfy the following conditions:

$\left(V_{3}\right) V$ is continuous differentiable and there exist positive constants $b_{3}>0, b_{4}>0$ such that $b_{3}|u|^{p} \leq-V(t, u) \leq b_{4}|u|^{p}$ for all $(t, u) \in[0, T] \times \mathbb{R}^{N} ;$ 
$\left(\mathrm{V}_{4}\right)-V(t, u) \leq-V_{u}(t, u) u$ for all $(t, u) \in[0, T] \times \mathbb{R}^{N}$;

$\left(\mathrm{G}_{1}\right)$ There exist $0<v<p, 0<\tau<p, b_{5}>0, b_{6} \geq 0$ such that $b_{6}-b_{5}|u|^{\nu} \leq G_{k}(u) \leq 0$ for all $u \in \mathbb{R}^{N} \backslash\{0\}$ and $k=1,2, \ldots, m$. Moreover, there exist $i \in\{1,2, \ldots, m\}$ and $b_{7}>0$, $b_{8}>0$ such that $G_{i}(u) \leq-b_{7}|u|^{\tau}$ for $|u| \leq b_{8}$.

Then problem $\left(P_{p, 0}\right)$ possesses at least one non-zero solution generated by impulses.

Example 1.5 Assume that $p=4, V(t, u)=-|u|^{4}, G_{1}(u)=-|u|^{3}, m=1, \tau=v=3$. All the conditions of Theorem 1.4 can be satisfied.

Remark 1.6 In [22, 23], the impulses are all superquadratic. However, when $p=2$, here we consider the case of subquadratic impulses. Moreover, since the impulses $G_{k}(u) \geq 0$ in [24], so our results are also different from those in [24].

Theorem 1.7 Assume that the following conditions hold:

$\left(\mathrm{G}_{2}\right)$ There exists $\theta>p$ such that $g_{k}(u) u \leq \theta G_{k}(u)<0$ for all $u \in \mathbb{R}^{N} \backslash\{0\}$ and $k=1,2, \ldots, m$;

$\left(\mathrm{V}_{3}^{\prime}\right) V$ is continuous differentiable and there exist positive constants $b>0,1<\gamma \leq p$ such that $b|u|^{\gamma} \leq-V(t, u)$ for all $(t, u) \in[0, T] \times \mathbb{R}^{N}$;

$\left(\mathrm{V}_{4}^{\prime}\right)$ There exists a constant $p \leq \varrho<\theta$ such that $-V(t, u) \leq-V_{u}(t, u) u \leq-\varrho V(t, u)$ for all $(t, u) \in[0, T] \times \mathbb{R}^{N}$.

Then problem $\left(P_{p, 0}\right)(1<p<4)$ possesses at least one non-zero solution generated by impulses.

Remark 1.8 When $p=2$, it is easy to see that $\left(\mathrm{V}_{3}^{\prime}\right),\left(\mathrm{V}_{4}^{\prime}\right)$ are weaker than $\left(\mathrm{V}_{1}\right),\left(\mathrm{V}_{2}\right)$. Therefore, Theorem 1.7 improves Theorem A.

Indeed, taking

$$
p=2, \quad V(t, u)=-|u|^{\gamma}-|u|^{\varsigma}, \quad 1<\gamma<\varsigma \leq 2, \quad G_{k}(u)=-|u|^{\theta} .
$$

All the conditions in Theorem 1.7 are satisfied, but conditions in Theorem A can not be satisfied.

Theorem 1.9 Assume that $\left(\mathrm{V}_{3}\right),\left(\mathrm{V}_{4}\right)$ hold and the following conditions are satisfied:

$\left(\mathrm{V}_{5}\right) V_{u}\left(t, u_{1}-u_{2}\right)=V_{u}\left(t, u_{1}\right)-V_{u}\left(t, u_{2}\right)$

$\left(\mathrm{G}_{3}\right) \max \left\{\lim _{\sup } \sup _{u \rightarrow 0} \frac{-G_{k}(u)}{|u|^{p}}, \lim \sup _{|u| \rightarrow+\infty} \frac{-G_{k}(u)}{|u|^{p}}\right\}<A$ for any $k \in\{1,2, \ldots, m\}, 0<m A C_{1}^{p}<$ $b_{3}^{\prime}$, where $b_{3}^{\prime}=\min \left\{\frac{1}{p}, b_{3}\right\}, C_{1}$ is a constant which will be defined in Section 2;

$\left(\mathrm{G}_{4}\right)$ There exists a constant vector $\xi=\left(\xi^{1}, \xi^{2}, \ldots, \xi^{N}\right) \in \mathbb{R}^{N} \backslash\{0\}$ such that $\sum_{k=1}^{m} G_{k}(\xi)<$ $\int_{0}^{T} V(t, \xi) d t$

(F $\left.F_{1}\right) \quad\left(F_{u}\left(t, u_{1}\right)-F_{u}\left(t, u_{2}\right), u_{1}-u_{2}\right) \geq 0$ for all $u_{1}, u_{2} \in \mathbb{R}^{N}$.

Then there exist $\delta_{0}>0, B>0$ such that, for each $\mu \in\left[0, \delta_{0}\right]$, problem $\left(P_{p, \mu}\right), p \geq 2$, possesses at least two solutions generated by impulses; moreover, their norms are less than $B$.

Remark 1.10 Compared with Theorem A, Theorem 1.9 deals with the multiple solutions generated by impulses. Moreover, we do not need $F_{u} \equiv 0$ as that in Theorem A. 
Example 1.11 Let $p=2$ and $V(t, u)=-|u|^{2}$. It is easy to see that conditions $\left(\mathrm{V}_{3}\right),\left(\mathrm{V}_{4}\right)$ and $\left(\mathrm{V}_{5}\right)$ hold. Let $T=1, m=1, \xi=(1,0, \ldots, 0)$ and

$$
G_{1}(u)= \begin{cases}-2|u|^{3}, & |u|<1 \\ -(6|u|-4), & |u| \geq 1 .\end{cases}
$$

Then we have

$$
\limsup _{u \rightarrow 0} \frac{-G_{1}(u)}{|u|^{2}}=\limsup _{|u| \rightarrow+\infty} \frac{-G_{1}(u)}{|u|^{2}}=0
$$

and

$$
-2=G_{1}(\xi)<\int_{0}^{T} V(t, \xi) d t=-1 .
$$

Hence, conditions $\left(\mathrm{G}_{3}\right)$ and $\left(\mathrm{G}_{4}\right)$ in Theorem 1.9 can be satisfied for sufficiently small $A>0$. Let $F(t, u)=u^{4}$, then it satisfies $\left(\mathrm{F}_{1}\right)$.

The paper is organized as follows. In Section 2, we present some preliminaries. In Section 3, we give the proof of our main results.

\section{Preliminaries}

In order to prove our main results, we give some definitions and lemmas that will be used in the proof of our main results. Let

$$
\begin{aligned}
H= & \left\{u:[0, T] \rightarrow \mathbb{R}^{N} \mid u\right. \text { is absolutely continuous, } \\
& \left.u(0)=u(T), u^{\prime} \in L^{p}\left([0, T], \mathbb{R}^{N}\right)\right\} .
\end{aligned}
$$

Then $H$ is a separable and reflexive Banach space with the norm defined by

$$
\|u\|=\left(\int_{0}^{T}\left[\left|u^{\prime}(t)\right|^{p}+|u(t)|^{p}\right] d t\right)^{1 / p}, \quad \forall u \in H .
$$

Let $\|\cdot\|_{p}=\left(\int_{0}^{T}|u(t)|^{p}\right)^{1 / p}$ denote the norm of Banach space of $L^{p}\left([0, T], \mathbb{R}^{N}\right)$. Since $(H,\|\cdot\|)$ is compactly embedded in $C\left([0, T], \mathbb{R}^{N}\right)$ (see [27]), we claim that there exists a positive constant $C_{1}$ such that

$$
\|u\|_{\infty} \leq C_{1}\|u\|
$$

where $\|u\|_{\infty}=\max _{t \in[0, T]}|u(t)|$.

To study problem $\left(P_{p, \mu}\right)$, we consider the functional $I$ defined by

$$
I(u)=\int_{0}^{T}\left[\frac{1}{p}\left|u^{\prime}\right|^{p}-V(t, u)+\mu F(t, u)\right] d t+\sum_{k=1}^{m} G_{k}\left(u\left(s_{k}\right)\right) .
$$

Similar to the proof of Lemma 1 of [24] (see also [20-22]), we can easily prove the following lemma. 
Lemma 2.1 Suppose $V, F \in C^{1}\left([0, T] \times \mathbb{R}^{N} \rightarrow \mathbb{R}\right), G_{k} \in C^{1}\left(\mathbb{R}^{N}, \mathbb{R}\right), k=1,2, \ldots, m$. Then $I$ is Frechét differentiable with

$$
I^{\prime}(u) v=\int_{0}^{T}\left[\left|u^{\prime}\right|^{p-2} u^{\prime} v^{\prime}-V_{u}(t, u) v+\mu F_{u}(t, u) v\right] d t+\sum_{k=1}^{m} g_{k}\left(u\left(s_{k}\right)\right) v\left(s_{k}\right)
$$

for any $u$ and $v$ in $H$. Furthermore, $u$ is a solution of $\left(P_{p, \mu}\right)$ if and only if $u$ is a critical point of I in $H$.

Lemma 2.2 $([27,28])$ Let $E$ be a real Banach space, and let $I \in C^{1}(E, \mathbb{R})$ satisfy the (P.S.) condition. Suppose $I(0)=0$ and

$\left(\mathrm{I}_{1}\right)$ there are constants $\rho, \beta>0$ such that $\left.I\right|_{\partial B_{\rho}} \geq \beta$, where $B_{\rho}=\{x \in E \mid\|x\|<\rho\}$,

$\left(\mathrm{I}_{2}\right)$ there is $e \in E \backslash B_{\rho}$ such that $I(e) \leq 0$.

Then I possesses a critical value $c \geq \beta$.

If $E$ is a real Banach space, denote by $\mathcal{W}_{X}$ (see [29]) the class of all functionals $\Phi: E \rightarrow$ $\mathbb{R}$ possessing the following property: if $\left\{u_{n}\right\}$ is a sequence in $E$ converging weakly to $u$ and $\liminf _{n \rightarrow \infty} \Phi\left(u_{n}\right) \leq \Phi(u)$, then $\left\{u_{n}\right\}$ has a subsequence converging strongly to $u$. For example, if $E$ is uniformly convex and $g:[0,+\infty) \rightarrow \mathbb{R}$ is a continuous, strictly increasing function, then, by classical results, the functional $u \rightarrow g(\|u\|)$ belongs to the class $\mathcal{W}_{X}$.

Lemma 2.3 ([29]) Let $E$ be a separable and reflexive real Banach space; let $\Phi: E \rightarrow \mathbb{R}$ be a coercive, sequentially weakly lower semicontinuous $C^{1}$ functional, belonging to $\mathcal{W}_{X}$, bounded on each bounded subset of $E$ and whose derivative admits a continuous inverse on $E^{*} ; J: E \rightarrow \mathbb{R}$ a $C^{1}$ functional with compact derivative. Assume that $\Phi$ has a strict local minimum $u_{0}$ with $\Phi\left(u_{0}\right)=J\left(u_{0}\right)=0$. Finally, setting

$$
\begin{aligned}
& \alpha^{\prime}=\max \left\{0, \limsup _{\|u\| \rightarrow+\infty} \frac{J(u)}{\Phi(u)}, \limsup _{u \rightarrow u_{0}} \frac{J(u)}{\Phi(u)}\right\} \\
& \beta^{\prime}=\sup _{u \in \Phi^{-1}(0,+\infty)} \frac{J(u)}{\Phi(u)},
\end{aligned}
$$

assume that $\alpha^{\prime}<\beta^{\prime}$. Then, for each compact interval $[a, b] \subset\left(\frac{1}{\beta^{\prime}}, \frac{1}{\alpha^{\prime}}\right)$ (with the conventions $\left.\frac{1}{0}=+\infty, \frac{1}{+\infty}=0\right)$, there exists $B>0$ with the following property: for every $\lambda \in[a, b]$ and every $C^{1}$, the functional $\Psi: E \rightarrow \mathbb{R}$ with compact derivative, there exists $\delta>0$ such that, for each $\mu \in[0, \delta]$, the equation

$$
\Phi^{\prime}(x)=\lambda J^{\prime}(x)+\mu \Psi^{\prime}(x)
$$

has at least three solutions in $E$ whose norms are less than $B$.

\section{Proof of the main results}

In this section, we give the proof of our main results in turn. $C_{i}, i=1,2, \ldots$, denotes different constants. 
Proof of Theorem 1.4 Since we consider problem $\left(P_{p, 0}\right)$, then $\mu \equiv 0$. In view of $\left(\mathrm{V}_{3}\right),\left(\mathrm{G}_{1}\right)$ and (2.3), we have

$$
\begin{aligned}
I(u) & =\int_{0}^{T}\left[\frac{1}{p}\left|u^{\prime}\right|^{p}-V(t, u)\right] d t+\sum_{k=1}^{m} G_{k}\left(u\left(s_{k}\right)\right) \\
& \geq \int_{0}^{T}\left[\frac{1}{p}\left|u^{\prime}\right|^{p}+b_{3}|u|^{p}\right] d t-b_{5} \sum_{k=1}^{m}\left|u\left(s_{k}\right)\right|^{\nu}+C_{2} \\
& \geq \min \left\{\frac{1}{p}, b_{3}\right\}\|u\|^{p}-C_{3}\|u\|_{\infty}^{\nu}+C_{2} \\
& \geq \min \left\{\frac{1}{p}, b_{3}\right\}\|u\|^{p}-C_{4}\|u\|^{v}+C_{2} .
\end{aligned}
$$

Since $v<p$, this implies that $\lim _{\|u\| \rightarrow \infty} I(u)=\infty$, i.e., $I$ is coercive.

If $\left\{u_{k}\right\}_{k \in \mathbb{N}} \subseteq H, u_{k} \rightarrow u$, then we have that $\left\{u_{k}\right\}_{k \in \mathbb{N}}$ converges uniformly to $u$ in $[0, T]$. By the continuity of $V, G_{k}$, we can easily know that $I$ is weakly lower semi-continuous. By Theorem 1.1 of [27], $I$ has a minimum point on $H$, which is a critical point of $I$.

In the following, we show that the critical point obtained above $u \neq 0$. Choose $v \in H$ such that $v\left(s_{i}\right) \neq 0$. By $\left(\mathrm{V}_{3}\right),\left(\mathrm{G}_{1}\right)$ and $(2.3)$, we have

$$
I(\xi v) \leq \xi^{p} \max \left\{\frac{1}{p}, b_{4}\right\}\|v\|^{p}-b_{7} \xi^{\tau}\left|v\left(s_{i}\right)\right|^{\tau}
$$

for $\xi>0$ small enough such that $\xi\left|v\left(s_{i}\right)\right| \leq b_{8}$. Since $\tau<p$, we can choose $\xi>0$ small enough such that $I(\xi v)<0$. This implies that the critical point obtained above is nontrivial.

Finally, we verify that the solution is generated by impulses. Suppose $g_{k} \equiv 0$ and $u$ is a solution for problem $\left(P_{p, 0}\right)$. By $(2.5),\left(\mathrm{V}_{3}\right),\left(\mathrm{V}_{4}\right)$, we have

$$
\begin{aligned}
0 & =\int_{0}^{T}\left[\left|u^{\prime}\right|^{p}-V_{u}(t, u) u\right] d t \\
& \geq \min \left\{1, b_{3}\right\}\|u\|^{p} .
\end{aligned}
$$

This means $u \equiv 0$. Therefore, problem $\left(P_{p, 0}\right)$ possesses at least one non-zero solution generated by impulses.

Proof of Theorem 1.7 We firstly show that $I$ satisfies the P.S. condition. Assume that $\left\{u_{j}\right\}_{j \in \mathbb{N}} \subset H$ is a sequence such that $\left\{I\left(u_{j}\right)\right\}_{j \in \mathbb{N}}$ is bounded and $I^{\prime}\left(u_{j}\right) \rightarrow 0$ as $j \rightarrow+\infty$. Then there exists a constant $C_{5}>0$ such that

$$
\left|I\left(u_{j}\right)\right| \leq C_{5}, \quad\left|I^{\prime}\left(u_{j}\right)\right| \leq C_{5}, \quad \forall j \in \mathbb{N} .
$$

By (3.1), (2.3), (2.4), (2.5), $\left(\mathrm{V}_{3}^{\prime}\right),\left(\mathrm{V}_{4}^{\prime}\right)$ and $\left(\mathrm{G}_{2}\right)$, we have

$$
\begin{aligned}
p C_{5}+C_{6}\left\|u_{j}\right\| & \geq p I\left(u_{j}\right)-\frac{p}{\theta} I^{\prime}\left(u_{j}\right) u_{j} \\
& =\left(1-\frac{p}{\theta}\right) \int_{0}^{T}\left|u_{j}^{\prime}\right|^{p} d t
\end{aligned}
$$




$$
\begin{aligned}
& +\int_{0}^{T}\left[\frac{p}{\theta} V_{u}\left(t, u_{j}\right) u_{j}-p V\left(t, u_{j}\right)\right] d t \\
& +p \sum_{k=1}^{m} G_{k}\left(u_{j}\left(s_{k}\right)\right)-\frac{p}{\theta} \sum_{k=1}^{m} g_{k}\left(u_{j}\left(s_{k}\right)\right) u_{j}\left(s_{k}\right) \\
\geq & \left(1-\frac{p}{\theta}\right) \int_{0}^{T}\left|u_{j}^{\prime}\right|^{p} d t+p\left(1-\frac{\varrho}{\theta}\right) \int_{0}^{T} b\left|u_{j}\right|^{\gamma} d t \\
\geq & \left(1-\frac{p}{\theta}\right) \int_{0}^{T}\left|u_{j}^{\prime}\right|^{p} d t \\
& +p\left(1-\frac{\varrho}{\theta}\right) b C_{1}^{\gamma-p}\left\|u_{j}\right\|^{\gamma-p} \int_{0}^{T}\left|u_{j}\right|^{p} d t \\
\geq & \min \left\{\left(1-\frac{p}{\theta}\right), p\left(1-\frac{\varrho}{\theta}\right) b C_{1}^{\gamma-p}\left\|u_{j}\right\|^{\gamma-p}\right\}\left\|u_{j}\right\|^{p} \\
\geq & \min \left\{\left(1-\frac{p}{\theta}\right)\left\|u_{j}\right\|^{p}, p\left(1-\frac{\varrho}{\theta}\right) b C_{1}^{\gamma-p}\left\|u_{j}\right\|^{\gamma}\right\} .
\end{aligned}
$$

It follows from (3.2) that $\left\{u_{j}\right\}_{j \in \mathbb{N}}$ is bounded in $H$. In a similar way to Lemma 3.1 in [30], we can prove that $\left\{u_{j}\right\}_{j \in \mathbb{N}}$ has a convergent subsequence in $H$. Hence, $I$ satisfies the P.S. condition.

Second, we verify $\left(\mathrm{I}_{1}\right)$ of Lemma 2.2. By $\left(\mathrm{G}_{2}\right)$ and (2.3), there exists $\delta_{1}>0$ such that for any $\|u\|_{\infty} \leq \delta_{1}$,

$$
\left|G_{k}(u)\right| \leq \frac{1}{4 m C_{1}}|u|^{p} .
$$

By (2.3), there exists $0<\delta<1$ such that for any $\|u\| \leq \delta$, the inequality $\|u\|_{\infty} \leq$ $\delta_{1}$ holds. Then, for $u \in H$ with $\|u\|=\delta_{0}$ and $1<p<4, \delta_{0}$ small enough $\left(0<\delta_{0}<\right.$ $\left.\min \left\{\left(p b C_{1}^{\gamma-p}\right)^{1 / p-\gamma}, \delta\right\}\right)$ such that

$$
\begin{aligned}
I(u) & \geq \frac{1}{p} \int_{0}^{T}\left|u^{\prime}(t)\right|^{p} d t+b \int_{0}^{T}|u(t)|^{\gamma} d t-\frac{1}{4 C_{1}}\|u\|_{\infty}^{p} \\
& \geq \min \left\{\frac{1}{p}\|u\|^{p}, b C_{1}^{\gamma-p}\|u\|^{\gamma}\right\}-\frac{1}{4}\|u\|^{p} \\
& \geq \frac{1}{p}\|u\|^{p}-\frac{1}{4}\|u\|^{p} \\
& \geq \frac{1}{4}\left\|\delta_{0}\right\|^{p} .
\end{aligned}
$$

Similar to [20] and [22], by $\left(\mathrm{V}_{4}^{\prime}\right)$, there exist $C_{7}, C_{8}>0$ such that

$$
-V(t, u) \leq C_{7}|u|^{\varrho}+C_{8} \quad \text { for all }(t, u) \in[0, T] \times \mathbb{R}^{N}
$$

By $\left(G_{2}\right)$, there exist $C_{9}, C_{10}>0$ such that

$$
G_{k}(u) \leq-C_{9}|u|^{\theta}+C_{10} \quad \text { for all } u \in \mathbb{R}^{N}, k \in\{1,2, \ldots, m\}
$$


To verify $\left(\mathrm{I}_{2}\right)$ of Lemma 2.2 , choose $v \in H$ such that $v\left(s_{k}\right) \neq 0$ for some $k \in\{1,2, \ldots, m\}$. Hence, we obtain

$$
I(\eta v) \leq \frac{\eta^{p}}{p} \int_{0}^{T}\left|v^{\prime}(t)\right|^{p} d t+C_{7}|\eta|^{\varrho} \int_{0}^{T}|v(t)|^{\varrho} d t-C_{9}|\eta|^{\theta} \sum_{k=1}^{m}\left|v\left(s_{k}\right)\right|^{\theta}+C_{11} .
$$

Since $\theta>p, \varrho \in[p, \theta)$, (3.7) implies $I(\eta v) \rightarrow-\infty$ as $\eta \rightarrow \infty$. So, for sufficiently large $\eta$, $e=\eta v$ satisfies condition $\left(\mathrm{I}_{2}\right)$. By the mountain pass lemma (Lemma 2.2), I possesses at least one non-zero critical point. Then, by Lemma 2.1, problem $\left(P_{p, 0}\right)$ has at least one non-zero solution.

Finally, we verify that the solution is generated by impulses. Suppose $g_{k} \equiv 0$ and $u$ is a solution for problem $\left(P_{p, 0}\right)$. Then, by $(2.5),\left(\mathrm{V}_{3}^{\prime}\right)$ and $\left(\mathrm{V}_{4}^{\prime}\right)$,

$$
\begin{aligned}
0 & =\int_{0}^{T}\left[\left|u^{\prime}\right|^{p}-V_{u}(t, u) u\right] d t \\
& \geq \int_{0}^{T}\left[\left|u^{\prime}\right|^{p}+b|u|^{\gamma}\right] d t \\
& \geq \int_{0}^{T}\left|u^{\prime}\right|^{p} d t+b\|u\|_{\infty}^{\gamma-p} \int_{0}^{T}|u|^{p} d t \\
& \geq \int_{0}^{T}\left|u^{\prime}\right|^{p} d t+b C_{1}^{\gamma-p}\|u\|^{\gamma-p} \int_{0}^{T}|u|^{p} d t \\
& \geq \min \left\{1, b C_{1}^{\gamma-p}\|u\|^{\gamma-p}\right\}\|u\|^{p} \\
& =\min \left\{\|u\|^{p}, b C_{1}^{\gamma-p}\|u\|^{\gamma}\right\} .
\end{aligned}
$$

This implies that $u \equiv 0$, i.e., problem $\left(P_{p, 0}\right)$ does not possess any non-zero solutions when impulses are zero. Hence, by Definition 1.1 or Definition 1.2, the non-zero solution obtained above is generated by impulses.

Proof of Theorem 1.9 In order to apply Lemma 2.3, we let

$$
\begin{aligned}
& \Phi(u)=\int_{0}^{T}\left[\frac{1}{p}\left|u^{\prime}(t)\right|^{p}-V(t, u(t))\right] d t, \\
& J(u)=-\sum_{k=1}^{m} G_{k}\left(u\left(s_{k}\right)\right), \quad \Psi(u)=-\int_{0}^{T} F(t, u(t)) d t .
\end{aligned}
$$

By Lemma 2.1, the critical points of the functional $\Phi-J-\mu \Psi$ are exactly the solutions for problem $\left(P_{p, \mu}\right)$. Hence, to prove our result, it is enough to apply Lemma 2.3 and show that we can choose $\lambda=1 \in[a, b] \subset\left(\frac{1}{\beta^{\prime}}, \frac{1}{\alpha^{\prime}}\right)$. We divide our proof into four steps as follows.

Step 1. We show that some fundamental assumptions are satisfied. Obviously, $E=H$ is a separable and uniformly convex Banach space. By $\left(\mathrm{V}_{3}\right)$, we have

$$
b_{3}^{\prime}\|u\|^{p} \leq \Phi(u) \leq b_{4}^{\prime}\|u\|^{p},
$$

where $b_{3}^{\prime}=\min \left\{\frac{1}{p}, b_{3}\right\}, b_{4}^{\prime}=\max \left\{\frac{1}{p}, b_{4}\right\}$, Hence, by (3.10) and Lemma 2.1, we can obtain that $\Phi(u)$ is a coercive $C^{1}$ functional bounded on each bounded subset of $E$. For a sequence $\left\{u_{n}\right\} \subset H$, if $u_{n} \rightarrow u \in H$ and $\liminf _{n \rightarrow \infty} \Phi\left(u_{n}\right) \leq \Phi(u)$, then $u_{n} \rightarrow u \in C[0, T]$. 
This means $\int_{0}^{T}\left[-\frac{1}{p}|u(t)|^{p}-V(t, u(t))\right] d t$ is weakly continuous. Hence, we have $\Phi(u)=$ $\frac{1}{p}\|u\|^{p}+\int_{0}^{T}\left[-\frac{1}{p}|u(t)|^{p}-V(t, u(t))\right] d t$ is sequentially weakly lower semicontinuous and $\liminf _{n \rightarrow \infty}\left\|u_{n}\right\|^{p} \leq\|u\|^{p}$. Therefore, $\left\{u_{n}\right\}$ has a subsequence converging strongly to $u$, i.e., $\Phi(u)$ belongs to $\mathcal{W}_{X}$. For any $u \in H \backslash\{0\}$, we have

$$
\begin{aligned}
\left\langle\Phi^{\prime}(u), u\right\rangle & =\int_{0}^{T}\left[\left(\left|u^{\prime}(t)\right|^{p-2} u^{\prime}(t), u^{\prime}(t)\right)-\left(V_{u}(t, u(t)), u(t)\right)\right] d t \\
& \geq \int_{0}^{T}\left[\left(\left|u^{\prime}(t)\right|^{p-2} u^{\prime}(t), u^{\prime}(t)\right)+b_{3}|u(t)|^{p}\right] d t \\
& \geq \min \left\{1, b_{3}\right\}\|u\|^{p} .
\end{aligned}
$$

So, $\lim _{\|u\| \rightarrow \infty}\left\langle\Phi^{\prime}(u), u\right\rangle /\|u\|=+\infty$, that is, $\Phi^{\prime}$ is coercive. Recall the following well-known inequality (see [30]): for any $x, y \in \mathbb{R}^{N}$, there exists a constant $C_{p}>0$ such that

$$
\left(|x|^{p-2} x-|y|^{p-2} y, x-y\right) \geq C_{p}|x-y|^{p}, \quad p \geq 2 .
$$

Hence, in view of $\left(\mathrm{V}_{3}\right)-\left(\mathrm{V}_{5}\right)$, for any $u, v \in H$, one has

$$
\begin{aligned}
\left\langle\Phi^{\prime}(u)-\Phi^{\prime}(v), u-v\right\rangle= & \int_{0}^{T}\left(\left|u^{\prime}(t)\right|^{p-2} u^{\prime}(t)-\left|v^{\prime}(t)\right|^{p-2} v^{\prime}(t), u^{\prime}(t)-v^{\prime}(t)\right) d t \\
& -\int_{0}^{T}\left(V_{u}(t, u(t))-V_{u}(t, v(t)), u(t)-v(t)\right) d t \\
\geq & C_{p} \int_{0}^{T}\left|u^{\prime}-v^{\prime}\right|^{p} d t \\
& +\int_{0}^{T} b_{3}|u(t)-v(t)|^{p} d t \\
\geq & \min \left\{C_{p}, b_{3}\right\}\|u-v\|^{p} .
\end{aligned}
$$

So, $\Phi^{\prime}$ is uniformly monotone. By [31, Theorem 26.A(d)], we have that $\left(\Phi^{\prime}\right)^{-1}$ exists and is continuous. For any $u, v \in H$,

$$
\left\langle J^{\prime}(u), v\right\rangle=-\sum_{k=1}^{m} g_{k}\left(u\left(s_{k}\right)\right) v\left(s_{k}\right), \quad\left\langle\Psi^{\prime}(u), v\right\rangle=-\int_{0}^{T} F_{u}(t, u(t)) v(t) d t
$$

Let $u_{n} \rightarrow u \in H$, then $u_{n} \rightarrow u \in C[0, T]$. Hence, $J^{\prime}\left(u_{n}\right) \rightarrow J^{\prime}(u), \Psi^{\prime}\left(u_{n}\right) \rightarrow \Psi^{\prime}(u)$ as $n \rightarrow \infty$. Therefore, we have $J^{\prime}, \Psi^{\prime}$ are compact operators by [31, Proposition 26.2]. In addition, $\Phi$ has a strict local minimum 0 with $\Phi(0)=J(0)=0$.

Step 2 . We show that $\alpha^{\prime}<1, \beta^{\prime}>1$. In view of $\left(G_{3}\right)$, there exist $0<\tau_{1}<\tau_{2}$ such that

$$
-G_{k}(u) \leq A|u|^{p}
$$

for any $|u| \in\left[0, \tau_{1}\right) \cup\left(\tau_{2},+\infty\right)$ and $k \in\{1,2, \ldots, m\}$. By the continuity of $G_{k},-G_{k}(u)$ is bounded for any $|u| \in\left[\tau_{2}, \tau_{1}\right]$. We can choose $C_{12}>0$ and $\sigma>p$ such that

$$
-G_{k}(u) \leq A|u|^{p}+C_{12}|u|^{\sigma}
$$


for any $u \in \mathbb{R}^{N}$. Hence, by (2.3), we have

$$
\begin{aligned}
J(u) & =-\sum_{k=1}^{m} G_{k}\left(u\left(s_{k}\right)\right) \\
& \leq \sum_{k=1}^{m}\left[A\left|u\left(s_{k}\right)\right|^{p}+C_{12}\left|u\left(s_{k}\right)\right|^{\sigma}\right] \\
& \leq m A\|u\|_{\infty}^{p}+C_{12} m\|u\|_{\infty}^{\sigma} \\
& \leq m A C_{1}^{p}\|u\|^{p}+m C_{12} C_{1}^{\sigma}\|u\|^{\sigma}
\end{aligned}
$$

for all $u \in H$. By (3.10) and (3.15), we obtain

$$
\limsup _{u \rightarrow 0} \frac{J(u)}{\Phi(u)} \leq \frac{m A C_{1}^{p}}{b_{3}^{\prime}}<1
$$

On the other hand, if $\left|u\left(s_{k}\right)\right| \leq \tau_{2}$, we have $-G_{k}\left(u\left(s_{k}\right)\right) \leq C_{13}$; if $\left|u\left(s_{k}\right)\right|>\tau_{2}$, we have $-G_{k}\left(u\left(s_{k}\right)\right) \leq A\left|u\left(s_{k}\right)\right|^{p}$. Then it follows that $-G_{k}\left(u\left(s_{k}\right)\right) \leq C_{13}+A\left|u\left(s_{k}\right)\right|^{p}$ for all $k \in$ $\{1,2, \ldots, m\}$. Hence, we obtain

$$
J(u) \leq C_{14}+m A C_{1}^{p}\|u\|^{p} .
$$

Therefore, we have

$$
\limsup _{\|u\| \rightarrow \infty} \frac{J(u)}{\Phi(u)} \leq \frac{m A C_{1}^{p}}{b_{3}^{\prime}}<1
$$

Combining (3.17) with (3.18), one has

$$
\alpha^{\prime}=\max \left\{0, \limsup _{\|u\| \rightarrow+\infty} \frac{J(u)}{\Phi(u)}, \limsup _{u \rightarrow u_{0}} \frac{J(u)}{\Phi(u)}\right\}<1 .
$$

By $\left(G_{4}\right)$, we obtain

$$
\beta^{\prime}=\sup _{u \in \Phi^{-1}(0,+\infty)} \frac{J(u)}{\Phi(u)} \geq \frac{-\sum_{k=1}^{m} G_{k}(\xi)}{-\int_{0}^{T} V(t, \xi) d t}>1
$$

Step 3. We show that problem $\left(P_{p, \mu}\right)$ has at least three solutions.

We can choose a compact interval $[a, b] \subset\left(\frac{1}{\beta^{\prime}}, \frac{1}{\alpha^{\prime}}\right)$ such that $\lambda=1 \in[a, b]$. By Lemma 2.3, there exists $B>0$ with the following property: for every $F$, there exists $\delta>0$ such that, for each $\mu \in[0, \delta]$, the equation

$$
\Phi^{\prime}(x)=J^{\prime}(x)+\mu \Psi^{\prime}(x)
$$

i.e., problem $\left(P_{p, \mu}\right), p \geq 2$, has at least three solutions in $E$ whose norms are less than $B$.

Step 3. We show that problem $\left(P_{p, \mu}\right)$ has at least two solutions generated by impulses. First, we verify that problem $\left(P_{p, \mu}\right), p \geq 2$, has at most one solution when $g_{k} \equiv 0$. On the 
contrary, assuming that problem $\left(P_{p, \mu}\right), p \geq 2$, has at least two distinct solutions $u_{1}, u_{2}$ and $g_{k} \equiv 0$, we have

$$
\begin{aligned}
0= & \left\langle I^{\prime}\left(u_{1}\right)-I^{\prime}\left(u_{2}\right), u_{1}-u_{2}\right\rangle \\
= & \int_{0}^{T}\left(\left|u_{1}^{\prime}(t)\right|^{p-2} u_{1}(t)-\left|u_{2}^{\prime}(t)\right|^{p-2} u_{2}(t), u_{1}^{\prime}(t)-u_{2}^{\prime}(t)\right) d t \\
& -\int_{0}^{T}\left(V_{u}\left(t, u_{1}(t)\right)-V_{u}\left(t, u_{2}(t)\right), u_{1}(t)-u_{2}(t)\right) d t \\
& +\mu \int_{0}^{T}\left(F_{u}\left(t, u_{1}(t)\right)-F_{u}\left(t, u_{2}(t)\right), u_{1}(t)-u_{2}(t)\right) d t \\
\geq & \min \left\{C_{p}, b_{3}\right\}\left\|u_{1}-u_{2}\right\|^{p} .
\end{aligned}
$$

Hence, we get $\left\|u_{1}-u_{2}\right\|=0$, i.e., problem $\left(P_{p, \mu}\right), p \geq 2$, has at most one solution when impulses are zero. By Remark 1.3, we can obtain that problem $\left(P_{p, \mu}\right), p \geq 2$, has at least two solutions generated by impulses.

\section{Competing interests}

The author declares that they have no competing interests.

\section{Acknowledgements}

This work was supported by the Natural Science Foundation of Hunan Province (12JJ9001), Hunan Provincial Science and Technology Department of Science and Technology Project (2012SK3117) and Construct Program of the key discipline in Hunan Province.

\section{Received: 7 May 2013 Accepted: 30 October 2013 Published: 02 Dec 2013}

\section{References}

1. George, PK, Nandakumaran, AK, Arapostathis, A: A note on controllability of impulsive systems. J. Math. Anal. Appl. 241, 276-283 (2000)

2. Carter, TE: Necessary and sufficient conditions for optimal impulsive rendezvous with linear equations of motion. Dyn. Control 10, 219-227 (2000)

3. Pei, Y, Li, C, Chen, L, Wang, C: Complex dynamics of one-prey multi-predator system with defensive ability of prey and impulsive biological control on predators. Adv. Complex Syst. 8, 483-495 (2005)

4. Liu, X, Willms, AR: Impulsive controllability of linear dynamical systems with applications to maneuvers of spacecraft. Math. Probl. Eng. 2, 277-299 (1996)

5. Gao, S, Chen, L, Nieto, JJ, Torres, A: Analysis of a delayed epidemic model with pulse vaccination and saturation incidence. Vaccine 24,6037-6045 (2006)

6. Chu, J, Nieto, JJ: Impulsive periodic solutions of first-order singular differential equations. Bull. Lond. Math. Soc. 40 143-150 (2008)

7. Zavalishchin, ST, Sesekin, AN: Dynamic Impulse System. Theory and Applications. Kluwer Academic, Dordrecht (1997)

8. Lakshmikantham, V, Bainov, DD, Simeonov, PS: Theory of Impulsive Differential Equations. World Scientific, Singapore (1989)

9. Haddad, WM, Chellaboina, C, Nersesov, SG, Sergey, G: Impulsive and Hybrid Dynamical Systems. Stability, Dissipativity and Control. Princeton University Press, Princeton (2006)

10. Samoilenko, AM, Perestyuk, NA: Impulsive Differential Equations. World Scientific, Singapore (1995)

11. Agarwal, RP, O'regan, D: A multiplicity result for second order impulsive differential equations via the Leffett Williams fixed point theorem. Appl. Math. Comput. 161, 433-439 (2005)

12. Wang, $\mathrm{H}$, Chen, $\mathrm{H}$ : Boundary value problem for second-order impulsive functional differential equations. Appl. Math. Comput. 191, 582-591 (2007)

13. Chen, J, Tisdel, CC, Yuan, R: On the solvability of periodic boundary value problems with impulse. J. Math. Anal. Appl. 331, 902-912 (2007)

14. Li, J, Nieto, JJ, Shen, J: Impulsive periodic boundary value problems of first-order differential equations. J. Math. Anal. Appl. 303, 288-303 (2005)

15. Hernandez, E, Henriquez, HR, Mckibben, MA: Existence results for abstract impulsive second-order neutral functional differential equations. Nonlinear Anal. TMA 70, 2736-2751 (2009)

16. Nieto, JJ, O'Regan, D: Variational approach to impulsive differential equations. Nonlinear Anal., Real World Appl. 10 680-690 (2009)

17. Tian, $Y, G e, W$ : Applications of variational methods to boundary value problem for impulsive differential equations. Proc. Edinb. Math. Soc. 51, 509-527 (2008) 
18. Zhang, Z, Yuan, R: An application of variational methods to Dirichlet boundary value problem with impulses. Nonlinear Anal., Real World Appl. 11, 155-162 (2010)

19. Zhou, J, Li, Y: Existence and multiplicity of solutions for some Dirichlet problems with impulsive effects. Nonlinear Anal. TMA 71, 2856-2865 (2009)

20. Sun, J, Chen, $\mathrm{H}$ : Variational method to the impulsive equation with Neumann boundary conditions. Bound. Value Probl. 2009, Article ID 316812 (2009)

21. Sun, J, Chen, H, Nieto, JJ, Otero-Novoa, M: The multiplicity of solutions for perturbed second-order Hamiltonian systems with impulsive effects. Nonlinear Anal. TMA 72, 4575-4586 (2010)

22. Zhang, H, Li, Z: Periodic and homoclinic solutions generated by impulses. Nonlinear Anal. 12(1), $39-51$ (2011)

23. Han, X, Zhang, H: Periodic and homoclinic solutions generated by impulses for asymptotically linear and sublinear Hamiltonian system. J. Comput. Appl. Math. 235, 1531-1541 (2011)

24. Han, X, Zhang, H, Li, Z: Periodic solutions for $p$-Laplacian systems with impulsive conditions. Math. Appl., 23(1), 213-218 (2010)

25. Teng, K, Zhang, C: Existence of solution to boundary value problem for impulsive differential equations. Nonlinear Anal., Real World Appl., 11(5), 4431-4441 (2010)

26. Zhang, L, Ge, W: Solvability of a kind of Sturm-Liouville boundary value problem with impulses via variational methods. Acta Appl. Math. 110, 1237-1248 (2010)

27. Mawhin, J, Willem, M: Critical Point Theory and Hamiltonian Systems. Springer, Berlin (1989)

28. Rabinowitz, PH: Minimax Methods in Critical Point Theory with Applications to Differential Equations. CBMS Reg. Conf. Ser. in Math., vol. 65. Am. Math. Soc., Providence (1986)

29. Ricceri, B: A further three critical points theorem. Nonlinear Anal. TMA 71, 4151-4157 (2009)

30. Teng, K: On variational methods to a generalized Emden-Fowler equation. Positivity 14, 335-351 (2010)

31. Zeidler, E: Nonlinear Functional Analysis and Its Applications, vol. 2. Springer, Berlin (1990)

10.1186/1687-1847-2013-347

Cite this article as: Yang: Existence and multiplicity of periodic solutions generated by impulses. Advances in Difference Equations 2013, 2013:347

\section{Submit your manuscript to a SpringerOpen ${ }^{0}$ journal and benefit from:}

- Convenient online submission

- Rigorous peer review

- Immediate publication on acceptance

Open access: articles freely available online

High visibility within the field

- Retaining the copyright to your article 